\title{
BMC Palliative Care reviewer acknowledgement 2015
}

Dirk Krüger

\section{Contributing reviewers}

The editors of BMC Palliative Care would like to thank all our reviewers who have contributed to the journal in Volume 14 (2015).

Aziz Ansari

USA

Abraham Brody

USA

Carole Gardner

UK

Ai Oishi

UK

Albert J Kirshen

Canada

Anthony Mancini

USA

Angela Kydd

UK

Anna Thit Johnsen

Denmark

Anne Hofmeyer

Australia

Anne Wilkinson

Australia

Arif Kamal

USA

Sue Ronaldson

Australia

Jane Phillips

Australia
Ben White

Australia

Melissa Bloomer

Australia

Gregory Crawford

Australia

Geoffrey Mitchell

Australia

Maryann Street

Australia

Gaye Moore

Australia

Anna Chur-Hansen

Australia

Petra Graham

Australia

Tim Luckett

Australia

Martin Tattersall

Australia

Adam Walczak

Australia

Sarah Jeong

Australia

Barbara Head

USA
Baukje Miedema

Anja Declercq

Belgium

Joachim Cohen

Belgium

Benjamin Cheng

Hong Kong

Patria Berry

USA

Bert Leysen

Belgium

Juliano Ferreira Arcuri

Brazil

Carlos Paiva

Brazil

David Brent

USA

Susan McClement

Canada

Christopher Klinger

Canada

Kimberley Widger

Canada

Ebru Kaya

Canada

Correspondence: dirk.krueger@biomedcentral.com

BioMed Central, Floor 6, 236 Gray's Inn Road, London WC1X 8HB, UK 


\begin{tabular}{|c|c|c|}
\hline $\begin{array}{l}\text { David Wright } \\
\text { Canada }\end{array}$ & $\begin{array}{l}\text { Christopher Klinger } \\
\text { Canada }\end{array}$ & $\begin{array}{l}\text { Miriam van Buiren } \\
\text { Germany }\end{array}$ \\
\hline $\begin{array}{l}\text { Breffni Hannon } \\
\text { Canada }\end{array}$ & $\begin{array}{l}\text { Claudia Gamondi } \\
\text { Switzerland }\end{array}$ & $\begin{array}{l}\text { Jan Gaertner } \\
\text { Germany }\end{array}$ \\
\hline $\begin{array}{l}\text { Lisa Barbera } \\
\text { Canada }\end{array}$ & $\begin{array}{l}\text { Cathy Campbell } \\
\text { USA }\end{array}$ & $\begin{array}{l}\text { Steffen T Simon } \\
\text { Germany }\end{array}$ \\
\hline $\begin{array}{l}\text { Krista Noonan } \\
\text { Canada }\end{array}$ & $\begin{array}{l}\text { Christopher Longo } \\
\text { Canada }\end{array}$ & $\begin{array}{l}\text { Klaus Maria Perrar } \\
\text { Germany }\end{array}$ \\
\hline $\begin{array}{l}\text { Tamara Sussman } \\
\text { Canada }\end{array}$ & $\begin{array}{l}\text { Christine McPherson } \\
\text { Canada }\end{array}$ & $\begin{array}{l}\text { Gloria Krahn } \\
\text { USA }\end{array}$ \\
\hline $\begin{array}{l}\text { Lorraine Venturato } \\
\text { Canada }\end{array}$ & $\begin{array}{l}\text { Massimo Costantini } \\
\text { Italy }\end{array}$ & $\begin{array}{l}\text { Graeme Rocker } \\
\text { Canada }\end{array}$ \\
\hline $\begin{array}{l}\text { Christopher De Bono } \\
\text { Canada }\end{array}$ & $\begin{array}{l}\text { David N Korones } \\
\text { USA }\end{array}$ & $\begin{array}{l}\text { Geoff Wong } \\
\text { USA }\end{array}$ \\
\hline $\begin{array}{l}\text { Genevieve Thompson } \\
\text { Canada }\end{array}$ & $\begin{array}{l}\text { Deborah Lewis } \\
\text { UK }\end{array}$ & $\begin{array}{l}\text { Hiroki Fukahori } \\
\text { Japan }\end{array}$ \\
\hline $\begin{array}{l}\text { Philippa Hawley } \\
\text { Canada }\end{array}$ & $\begin{array}{l}\text { Donna Wilson } \\
\text { Canada }\end{array}$ & $\begin{array}{l}\text { Andy Hau Yan Ho } \\
\text { Hong Kong }\end{array}$ \\
\hline Robin Cohen & $\begin{array}{l}\text { Donnamaria Cortezzo } \\
\text { USA }\end{array}$ & $\begin{array}{l}\text { Hon Wai Benjamin Cheng } \\
\text { Hong Kong }\end{array}$ \\
\hline Vera Hirsh & $\begin{array}{l}\text { Doralina Anghelescu } \\
\text { USA }\end{array}$ & $\begin{array}{l}\text { Iwao Osaka } \\
\text { Japan }\end{array}$ \\
\hline David Streiner & $\begin{array}{l}\text { Eleni Karasouli } \\
\text { UK }\end{array}$ & $\begin{array}{l}\text { Anthony Staines } \\
\text { Ireland }\end{array}$ \\
\hline Canada & Eloise Carr & Charles Normand \\
\hline Raymond Viola & Canada & Ireland \\
\hline Canada & Elaine Morgan & Claire Donnellan \\
\hline Harold Siden & USA & Ireland \\
\hline Canada & Felicity Hasson & Cinzia Brunelli \\
\hline Kevin Weingarten & UK & Italy \\
\hline Canada & $\begin{array}{l}\text { Fredrick Ashbury } \\
\text { Canada }\end{array}$ & $\begin{array}{l}\text { Paolo Gritti } \\
\text { Italy }\end{array}$ \\
\hline Canada & $\begin{array}{l}\text { Marilene Filbet } \\
\text { France }\end{array}$ & $\begin{array}{l}\text { Jan Schuling } \\
\text { Netherlands }\end{array}$ \\
\hline $\begin{array}{l}\text { Carmen Houben } \\
\text { Netherlands }\end{array}$ & $\begin{array}{l}\text { Freda Dekeyser Ganz } \\
\text { Israel }\end{array}$ & $\begin{array}{l}\text { Mitsunori Miyashita } \\
\text { Japan }\end{array}$ \\
\hline $\begin{array}{l}\text { Catherine Lam } \\
\text { USA }\end{array}$ & $\begin{array}{l}\text { Bruno Gagnon } \\
\text { Canada }\end{array}$ & $\begin{array}{l}\text { Hiroya Kinoshita } \\
\text { Japan }\end{array}$ \\
\hline $\begin{array}{l}\text { Catriona Kennedy } \\
\text { Ireland }\end{array}$ & $\begin{array}{l}\text { Jürgen in der Schmitten } \\
\text { Germany }\end{array}$ & $\begin{array}{l}\text { Nobuhisa Nakajima } \\
\text { Japan }\end{array}$ \\
\hline $\begin{array}{l}\text { Cheryl Nekolaichuk } \\
\text { Canada }\end{array}$ & $\begin{array}{l}\text { Waldemar Siemens } \\
\text { Germany }\end{array}$ & $\begin{array}{l}\text { Takuya Shinjo } \\
\text { Japan }\end{array}$ \\
\hline $\begin{array}{l}\text { Christian Schulz } \\
\text { Germany }\end{array}$ & $\begin{array}{l}\text { Claudia Bausewein } \\
\text { Germany }\end{array}$ & $\begin{array}{l}\text { João Batista Santos Garcia } \\
\text { Brazil }\end{array}$ \\
\hline
\end{tabular}


Jayne Brown

UK

Garcia Jesusangel

Spain

Joe Low

UK

Karin Oechsle

Germany

Karen Detering

Australia

Katherine Peters

USA

Kathi Mooney

USA

Moens Katrien

UK

Kazuki Sato

Japan

Kirsten Wentlandt

Canada

Kathrin Milbury

USA

Lenzo Robijn

Belgium

Lorraine Holtslander

Canada

Laura Gilchrist

USA

Marike De Boer

Netherlands

Maho Aoyama

Japan

Maria Arantzamendi

Spain

Sandra Pereira

Portugal

Maxwell Vergo

USA

Marilyn Ballantyne

Canada

Melanie Smith

USA

Melissa Garrido

USA
Melissa Henry

Canada

Michael Teut

Germany

Michael McCrory

USA

Margaret O'Connor

Australia

Marc Sampedro Pilegaard

Denmark

Nicholas Hughes

UK

Natalie Evans

Netherlands

Martin Smalbrugge

Netherlands

Eleni Volakli

Netherlands

Annicka van

Netherlands

Anneke Leijntje Francke

Netherlands

Nick Hex

UK

Arnstein Finset

Norway

Peter Hudson

Australia

Anna Rahman

USA

Richard Harding

UK

Robin Ray

Australia

Susan Elizabeth

UK

Sallie-Anne Pearson

Australia

Sandra Schellinger

USA

Sarah Goodlin

USA

Saskia Juenger

Germany
Antonio Noguera

Spain

Srini Chary

Canada

Sumit Gupta

USA

Susie Lund

UK

Hans Thulesius

Sweden

Claudia Gambdi

Switzerland

Sriram Yennu

USA

Tessa Watts

UK

Hsiao-Ting Chang

Taiwan

Wen-Chi Chou

Taiwan

Tak Fung

Canada

Thomas Carroll

USA

Sema Sezgin Goksu

Turkey

Julia Downing

Uganda

Richard Powell

Uganda

Katherine Bristowe

UK

Ellen Henderson

UK

Libby Sallnow

UK

Helen Kerr

UK

Susan Ashton

UK

Jo Hockley

UK

Mark Howard

UK 
Bob Whorton

UK

Victoria Allgar

UK

Padmanabhan Ramnarayan

UK

Caroline Barry

UK

Daniel Knights

UK

Victoria Simms

UK

Amy Gadoud

UK

Miguel Goncalves

UK

Paddy Stone

UK

Natalia Calanzani

UK

Sara Booth

UK

Mona Kanaan

UK

Olinda Santin

UK

Lorna Fraser

UK

Cara Bailey

UK

Charlotte Wilson

UK

Kristian Pollock

UK

Miranda Stacpoole UK
Nathan Davies

UK

Chloe Chin

UK

Richella Ryan

UK

Wei Gao

UK

Jan Fish

UK

Sandra Varey

UK

Liz Bryan

UK

Sarah Yardley

UK

Neil McHugh

UK

Ruth Diver

UK

Irene Tuffrey-Wijne

UK

Nwamaka Eneanya

USA

Maxine De La Cruz

USA

Deborah Carr

USA

Sriram Yennu

USA

Joshua Jones

USA

Sherika Newman

USA

Eric Widera

USA
Timothy DeEulis

USA

Kelly Michelson

USA

Joshua Hauser

USA

Ladislav Volicer

USA

Max Vergo

USA

Linda Emanuel

USA

M. Jeanne Wirpsa

USA

Thomas Carroll

USA

Marcy Rosenbaum

USA

Susan Enguidanos

USA

Egidio Del Fabbro

USA

Thomas Leblanc

USA

Elaine Wittenberg USA

Akhila Reddy

USA

Catherine Lam

USA

Elissa Miller

USA

Viv Lucas

UK 